\title{
Experimental Research on Internal Behaviors of Caved Rocks under the Uniaxial Confined Compression
}

\author{
Yu-jiang Zhang, ${ }^{1,2}$ Guo-rui Feng, ${ }^{1,2}$ and Ting-ye $\mathbf{Q i}^{2,3}$ \\ ${ }^{1}$ College of Mining Engineering, Taiyuan University of Technology, Taiyuan 030024, China \\ ${ }^{2}$ Shanxi Engineering Research Center for Green Mining, Taiyuan 030024, China \\ ${ }^{3}$ Institute of Mining Technology, Taiyuan University of Technology, Taiyuan 030024, China \\ Correspondence should be addressed to Guo-rui Feng; fguorui@163.com
}

Received 14 November 2016; Accepted 4 January 2017; Published 26 January 2017

Academic Editor: Fernando Lusquiños

Copyright (C) 2017 Yu-jiang Zhang et al. This is an open access article distributed under the Creative Commons Attribution License, which permits unrestricted use, distribution, and reproduction in any medium, provided the original work is properly cited.

\begin{abstract}
As main composition of longwall gob, caved rocks' behaviors and their impacts under compression crucially influence strata control, subsidence, associated resources extraction, and many other aspects. However, current researches are based on a whole sample, due to looseness of caved rocks and limitation of observation technology. In this paper, an experiment system was built to investigate internal behaviors of caved rocks' sample, under the uniaxial confined compression, including movement and breakage behavior by the digital image processing technologies. The results show that the compression process of caved rocks could be divided into two stages by relative density. Boundary effect and changes of voids and contact pressure among caved rocks lead to different movement law in different position in sample's interior. A stratification phenomenon of breakage was discovered, which presents breakage concentration in the middle of the sample. The nonlinear movement and shear dislocation induced by shifts among caved rocks are the reason of the breakage stratification phenomenon. This phenomenon would have an effect on the permeability and seepage research of similar medium.
\end{abstract}

\section{Introduction}

Longwall gob is composed of fractured and caved (or crushed) rocks and compressed by overburden load [1-3]. Considering the importance of caved rocks in longwall gob, they have been researched and applied in many respects, including inferring the position of main roof and overburden structure [4-6], estimating the pressure and permeability distribution of gob $[7,8]$, and evaluating feasibilities of mining in methane relieving seam [9]. In addition, compaction and seepage characteristics of caved rocks also are the basis of study on gas migration, enrichment, and extraction in gob [10-12]. However, comparing with researches of the whole sample composed of caved rocks under compression, little attention was paid to behaviors of sample's interior and their influences, due to looseness and irregularity of the caved rocks and limitation of observation technology. Thence, it is important for above researches to investigate internal behaviors of the whole sample under compression.
Laboratory tests of caved rocks under compression have been done, because the inaccessibility of the gob leads to difficulties of measurement in situ. Test samples are given though a scaled-down gradation curve which is from the actual gradation curve of gob materials [1, 10-13]. And uniaxial confined, biaxial, and triaxial compression tests were used to research compression behaviors, void characteristics, deformation and breakage properties, and secant and tangent modulus of caved rocks and similar materials [1, 13-15]. Moreover, the fractal theory has been used to study longwall goaf porosity and permeability distribution [10], the seepage theory has been used to study permeability characteristics under uniaxial compression using water flow $[11,12]$, and the diffusion theory has been used to study $\mathrm{Co}_{2}$ and $\mathrm{N}_{2}$ for the influence of gas migration rule [3]. However, these studies of caved rocks paid more attention to characteristics and rules of the whole specimen and ignored the internal behavior for these laws' impact, because the behaviors and their effects of among rock pieces are difficult to observe, due to looseness 

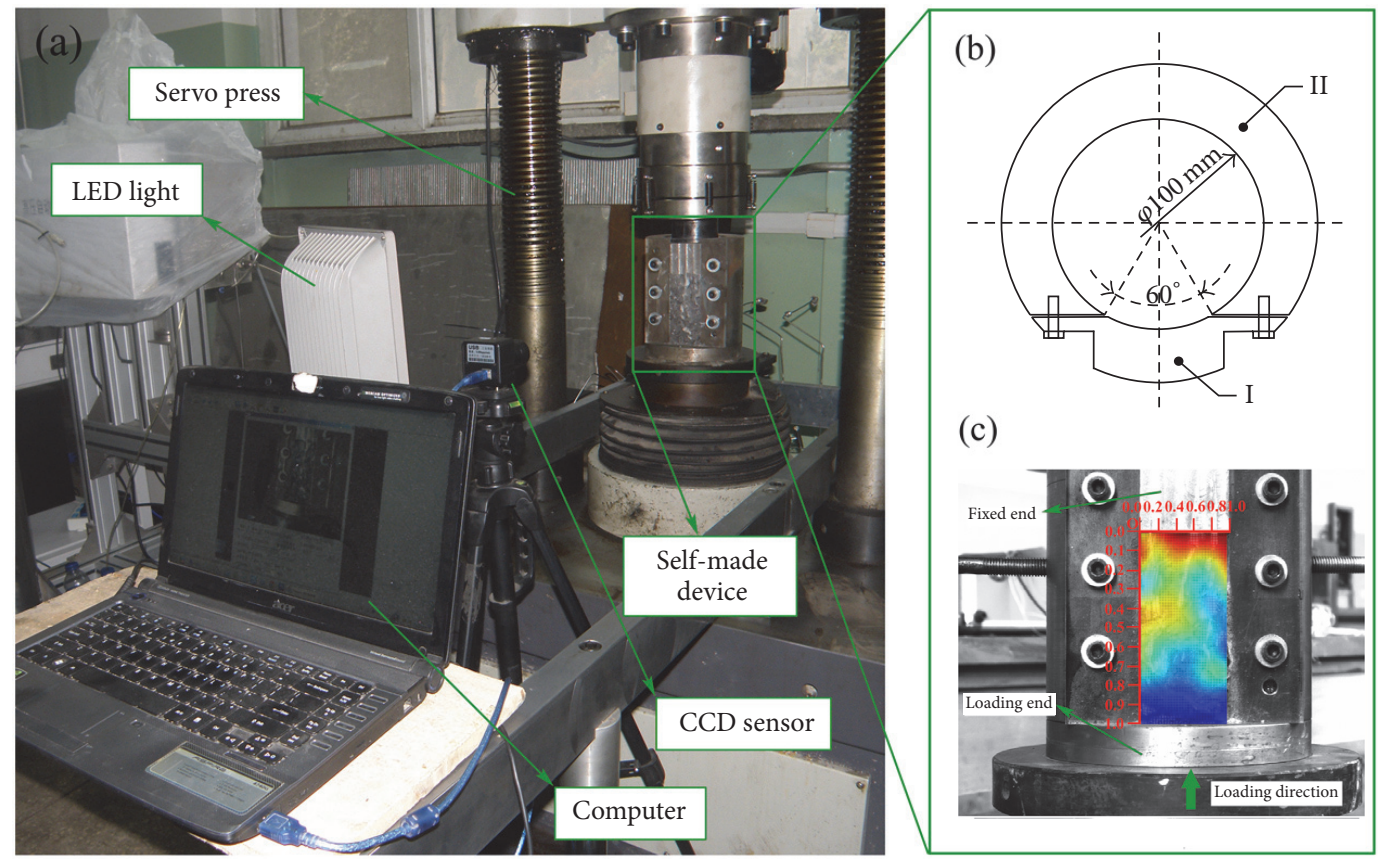

Figure 1: Test system.

of caved rocks and the limitation of observation technology and equipment material.

Numerical simulation method can overcome this deficiency of experiment to some extent [16-21]. However, there are still difficult in the description of irregular rock pieces and failure criteria, when using numerical simulation method. With the help of the microcomputed tomography (Micro-CT) or nuclear magnetic resonance (NMR), threedimensional reconstruction researches of porous media on the mesoscopic scale using numerical method have made great progress [22-25]. However, experiment of caved rocks required larger size and pressure cannot use the above methods, because of the limitation of observation equipment, loading device materials, and sample size. In recent years, with the development of computer technology and measurement technology, the digital image processing technologies have been widely used in the research of rock mechanics and mining [26-30]. Among them, the digital image correlation (DIC) technology and the machine vision could realize the real-time tracking of the rock pieces and provide a technique for experimental study on the internal behaviors of caved rocks and similar medium.

According to the above problem, self-made visual compression device was designed as a part of test system to observe behaviors of sample's interior by the digital image processing technologies. The test system, test sample, and testing procedure are introduced in Section 2. Moreover, in Section 3, we discuss the compression process and its stage division of caved rocks under the uniaxial confined compression, the behaviors of sample's interior including movement and breakage behaviors along the loading direction, and possible effects for seepage researches of gob and similar medium.

\section{Experiment}

2.1. Test System. A test system was established to record the behaviors of sample's interior under compression. As shown in Figure 1, the test system includes three main parts: servocontrolled testing machine, self-made device, and recording system composed of CCD sensor, LED light, and the computer. Among them, the self-made device is used to fill with caved rocks and the cylinder of the self-made device is divided into two parts (part I and part II) along the two generatrices (Figure 1(b)). A cross section of $170 \mathrm{~mm} * 50 \mathrm{~mm}$ in the side wall of part II is the observation window (Figure 1(c)). Part I could be replaced by an organic glass plate of $15 \mathrm{~mm}$ thick, when the behaviors of sample's interior need to be observed. The CCD sensor takes photos of the caved rocks though the observation window. The CCD sensor has $2560 \times$ 1920 pixels (5 megapixels), and its focal length is in the range of $6 \sim 12 \mathrm{~mm}$. The photos taken by the CCD sensor were stored in the computer.

2.2. Test Sample. In this study, caved rocks were collected from the gob of Number 8 coal seam in Baijiazhuang coal mine in Shanxi province, which has the average uniaxial compressive strength of $67.3 \mathrm{MPa}$, the average tensile strength of $2.47 \mathrm{MPa}$, and the average density of $2.96 \mathrm{~g} / \mathrm{cm}^{3}$. The caved rocks were crushed and separated into five groups with the particle size of $2.5-5 \mathrm{~mm}, 5-10 \mathrm{~mm}, 10-15 \mathrm{~mm}, 15-20 \mathrm{~mm}$, and $20-25 \mathrm{~mm}$, and these sizes have been used widely in research of gob material $[3,11,13]$. According to the gradation curve $[1,10]$ shown in Figure 2, the sample was constituted by mixture of different particle size. These gradation curves are scaled-down gradation curves according to the actual gradation curve of caved rocks and have negligible influence on the 


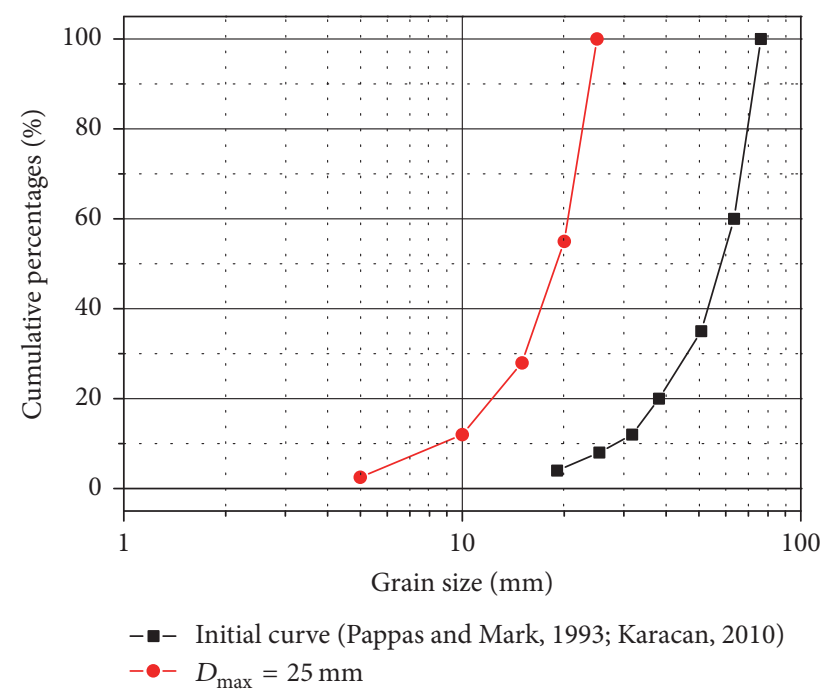

FIGURE 2: Grain size distribution curve of caved rocks.

result of the experiment $[1,10,31]$. Four groups experiments were conducted including one control group, using part I to replace organic glass.

2.3. Test Procedure. According to the gradation curve, caved rocks of different sizes were weighed accurately and mixed sufficiently. The mixed caved rocks were poured into the lubricated self-made device. After a slight shake, the indenter was put into the cylinder. The self-made device filled with mixed caved rocks and indenter were put on the centre of the platen. Then, arrange the CCD sensor and the LED light, and adjust the sensor's focal length and aperture. After that, the sample was loaded from the bottom up with $0.001 \mathrm{kN} / \mathrm{s}$. Meanwhile, the sample was simultaneously observed by taking photos using the CCD sensor, and the time interval for taking a photo was 4 seconds. The equivalent grain sizes and the number of rock pieces were extracted from photos by the machine vision [29]. The displacement was extracted by DIC technology, the basic principle and applications of which have been introduced in detail [32-34]. The ratio of real length and picture size is $0.099 \mathrm{~mm} / \mathrm{pixel}$, and the displacement measurement accuracy in this test is $1 \mu \mathrm{m}$ by using the subpixel algorithm (0.01 pixel) and calibration. In Figure 1(c), the picture shows a displacement contour which was processed by the DIC technology. The upper indenter is the fixed end and the bottom moves up as the loading end. The loading direction is from the bottom up. In order to describe the results more clearly, a reference frame was built in Figure 1(c), axes in which show the internal location of sample.

\section{Results and Discussions}

3.1. Stage Division of Compression. A stress-strain curve and displacement contours of it under some certain pressures were shown in Figure 3. The displacement contours were given by DIC technology and show the displacement of sample's interior along the loading direction. The corresponding pressures (shown in Figure 3 with two green points) of contours are $2.0 \mathrm{MPa}$ and $8.4 \mathrm{MPa}$ respectively.
As shown in Figure 3, the stress-strain curve of sample of caved rocks presents a nonlinear characteristic, and it still fits with Salamon's model under current pressure $[1,8]$. Under the same pressures, there are greater strain values than the common uniaxial confined compression, when the compression stress exceeds $6 \mathrm{MPa}$. A small stiffness of the organic glass plate compared to part I of the self-made device results in the situation. However, under the current pressure, the curve still fits with Salamon's model.

The compression process could be divided into two stages according to the trend of the stress-strain curve and breakage [35]. The main characteristics of the two stages are different, although void decrease and breakage both existed. In the first stage, the curve presents linear trend with the sliding and rotation of caved rock and the void decrease. After entering the second stage, caved rocks began to break under an enlarged pressure and the curve presents a nonlinear feature.

In the first stage, caved rocks overcome friction and move to a more stable position under the pressure. The main behaviors are decreases of void volume and porosity in the sample. Before entering the second stage, the caved rocks are in the most compact state [36]. Thence, the relative density could be used to be divided into two stages through the following formula [35]:

$$
D_{r}=\frac{e_{\max }-e}{e_{\max }-e_{\min }}
$$

where $D_{r}$ was the relative density; $e$ was the void ratio, $e=$ $\Phi /(1-\Phi), \Phi$ was porosity which was given by $[1] ; e_{\max }$ was the maximum void ratio; and $e_{\min }$ was minimum void ratio which was $35.04 \%$ calculated according to the dense packing theory [35].

According to [35], the range of $D_{r}$ is $0.67 \sim 1.0$ in the most compact state. The corresponding porosity and compression pressure are the range of $0.259-0.296$ and $3.1-6.7 \mathrm{MPa}$, respectively.

In Figure 3, displacement contours present a different trend in sample's interior between two stages. In order to reveal the phenomenon, the movement law along the loading direction was researched in the following study.

3.2. Movement of Sample's Interior. Figure 4 shows the cumulative displacements of different position in sample's interior. And the displacements of different positions, which are 0.14 , $0.3,0.46,0.65,0.89$, and 1 (it means loading end) according to the reference frame in Figure 1, were described in Figure 4. The $x$-axis and $y$-axis present the compression pressure and cumulative displacement along the loading direction. Only the displacements before $8.4 \mathrm{MPa}$ were given due to blurred images after that in Figure 4.

As shown in Figure 4, the movement trend of sample' interior related to its positon along the loading direction. With the increase of compression pressure, the cumulative displacement curves of different positions show the trend from centralizing to scattering. And the curvature degree of these curves increases with the distance decrease to fixed end. The curve (0.14) adjacent to the fixed end is different from 


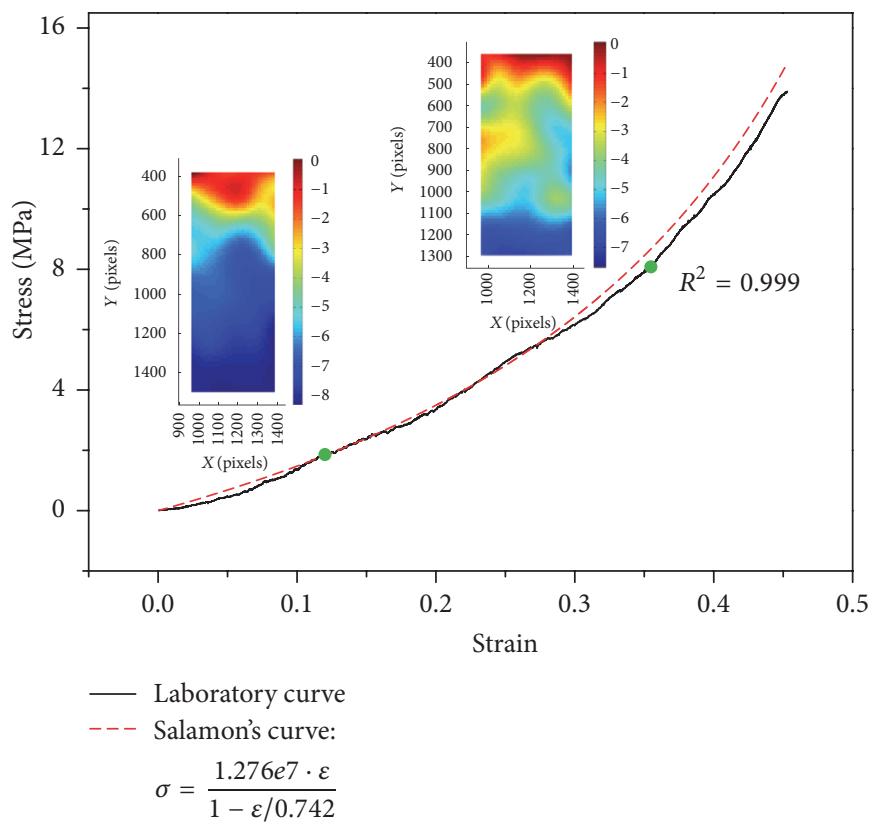

FIGURE 3: Stress-strain curve and displacement contours of it under some certain pressures.

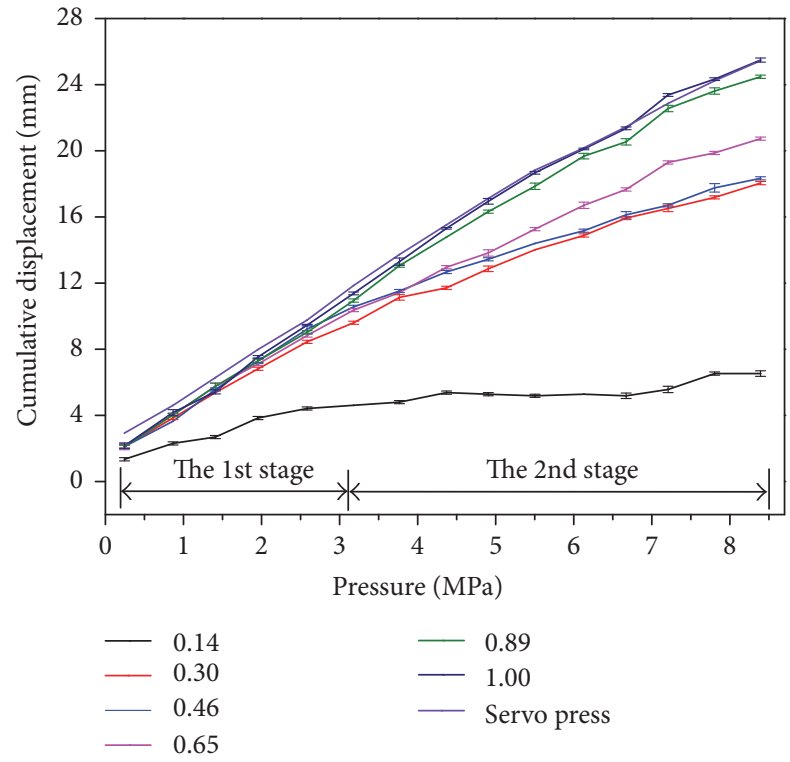

FIGURE 4: Relationship between cumulative displacement of different position and pressure.

others, which has a smaller displacement. In addition, in the first stage, the other curves are intensive. That means the small displacement differences among different positions in this stage. After that, the curves near the loading end and middle curves have a separation, which presents the enlargements of displacement differences between different positions.

According to Figure 4, the cumulative displacements of different position under the same pressure were given in Figure 5. And Figures 5(a) and 5(b) describe the displacements in the first stage and the second stage, respectively.
As shown in Figure 5, the displacement of sample' interior could be divided into section I and section II along the loading direction according to the shapes and trends of the curves. The change trends are different between the two stages.

In Figure 5(a), the displacements sharply decrease with the distance decreased to the fixed end in section I in the first stage, the trend of which is similar with the second stage. In section II, the displacement curves from about 0.3 to 1.0 are approximate straight lines in the first stage. This means the same displacement under the compression. In Figure 5(b), the displacement curves present new trends in section II which are different from ones in the first stage. And section II was divided into sections IIa and IIb through the displacement difference. The displacement differences between section IIa and section IIb began to enlarge in the second stage. That means a velocity difference along the loading direction in the sample's interior.

Relative displacement is defined as the relative position changes of some point along the loading direction between the initial and a given time in this paper. It presents compression degree in different positon along the loading direction. The positons of peak have maximal compression degree.

Figure 6 shows the changes of relative displacement under the compression. In Figure 6, the maximal relative displacement appears in the middle of the sample first, and it moves from the middle to the fixed end with the increase of pressure. That suggests the movement of maximal compression region.

Boundaries of the fixed end and the changes of voids and contact pressure result in the different displacement among the different position. In section I, the movable space is limited by the fixed end, even though there were the large void and small contact pressure in the first stage. Thence, the boundary effect of the fixed end as the dominant factor leads to the movement behaviors in section I. In section II, as far 


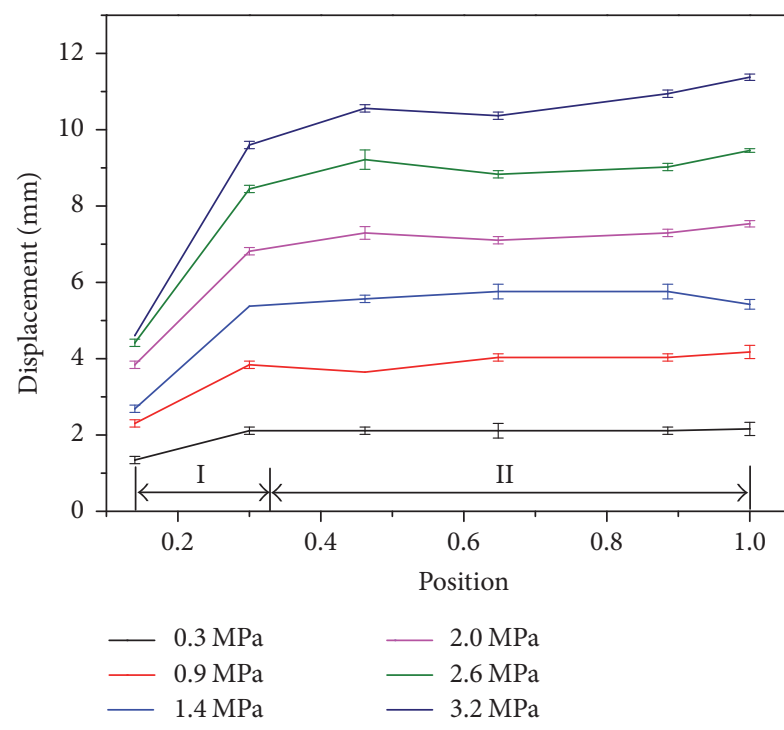

(a)

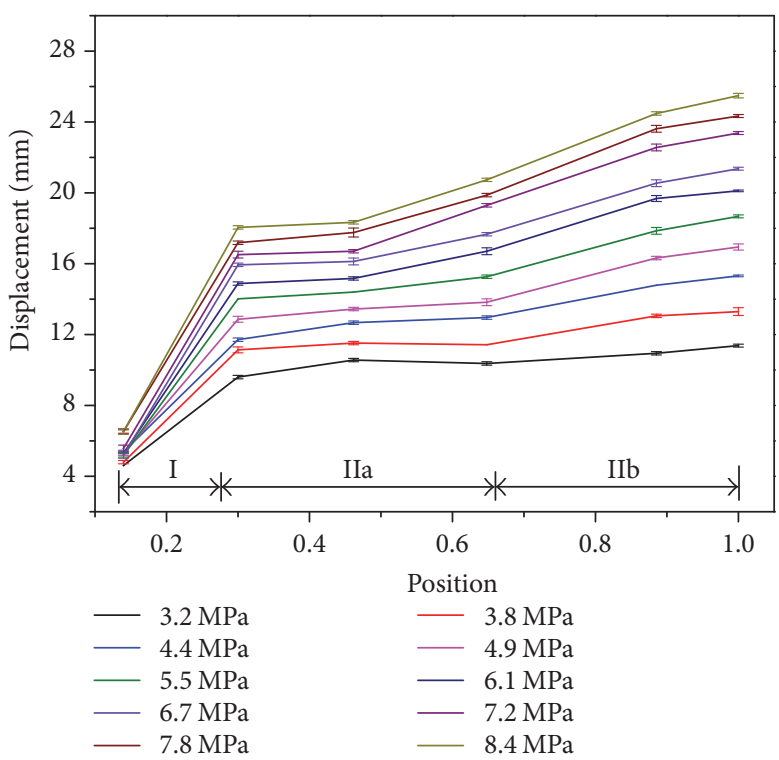

(b)

Figure 5: Cumulative displacement of different position of two stages.

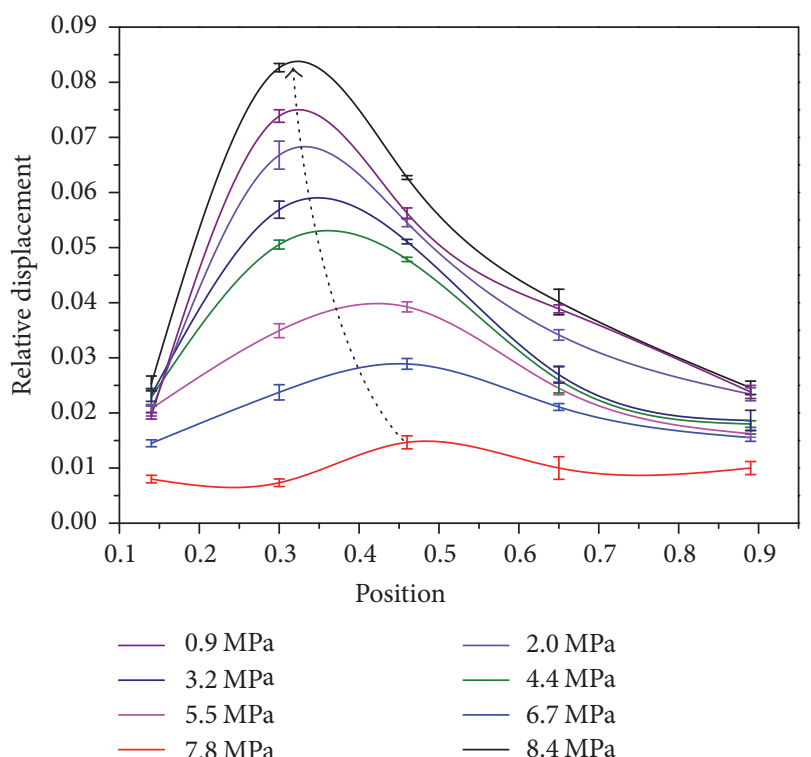

FIGURE 6: Relationship between position and relative displacement.

away from the fixed end, the boundary effect weakens and the changes of voids and contact pressure are the dominant factor in section II.

3.3. Stratification of Breakage. After entering the second stage, some large particle caved rocks began to fracture. However, an interesting phenomenon was discovered during the test, which is the concentrated distribution rather than uneven distribution of breakage in sample's interior (binary images in Figure 7). In order to quantitative analyze of this phenomenon, the pictures were chosen in equal intervals of time and in which the equivalent particle size and the number

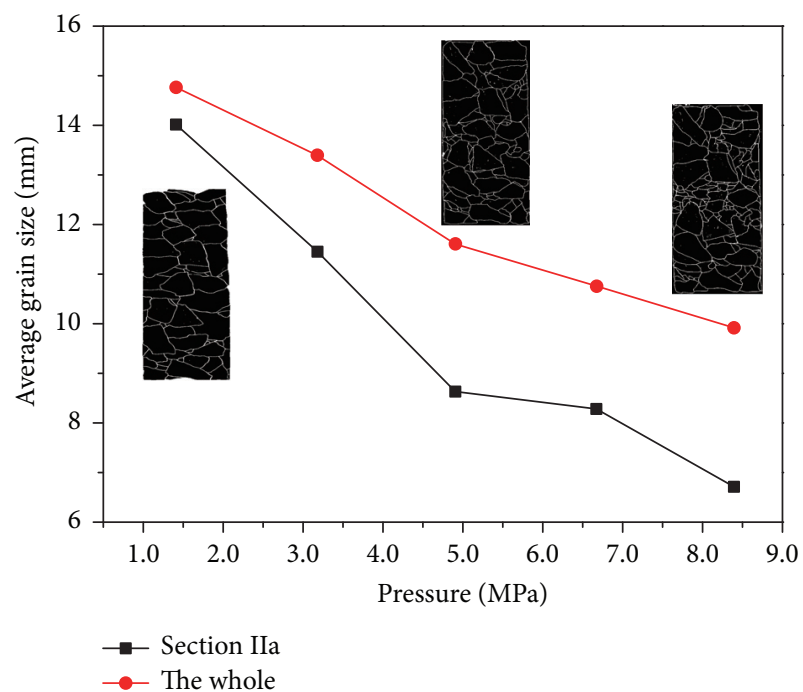

FIGURE 7: Average grain sizes of the whole and the section IIa and breakage situation.

of rock pieces were extracted [37]. Then, the average particle size of each picture was calculated by the equivalent particle size and number of rock pieces and the following formula [37]:

$$
D_{n L}=\sum_{i=1}^{n} \frac{n_{i} d_{i}}{n_{1}+n_{2}+\cdots n_{i} \cdots+n_{n}},
$$

where $d_{i}$ and $n_{i}$ are the average particle size and the corresponding number, respectively, of some group and $i$ is the group.

As shown in Figure 7, caved rocks in the middle (the range of $0.25-0.65$ ) of sample begin to break first with the 


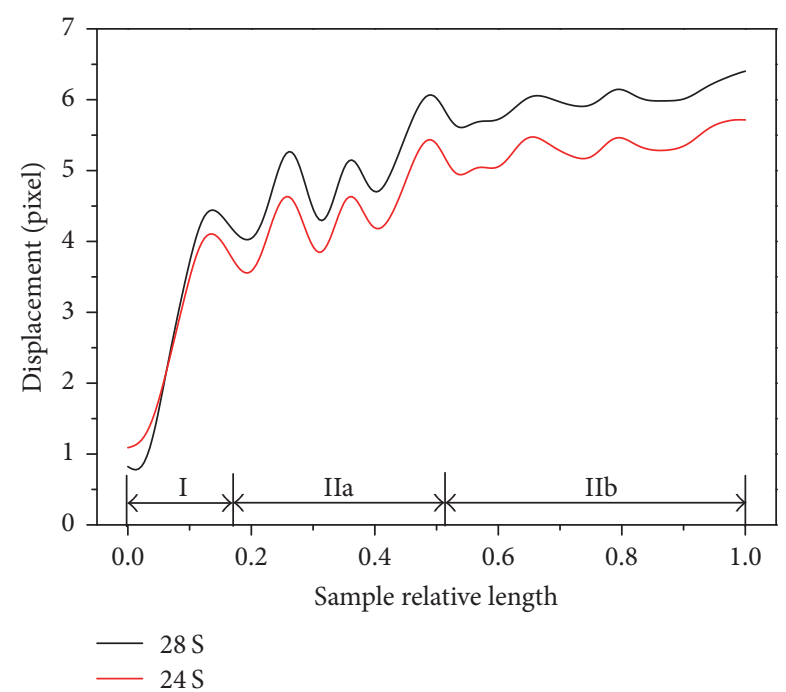

FIGURE 8: Displacement distribution in short time interval.

increase of compression pressure, and the breakage degree in the middle is greater than that at both ends. The average grain sizes of caved rocks of both the whole and section IIa of the sample decrease with the increasing pressure. However, the average grain sizes are smaller and their reduced rate is faster in section IIa than the whole. All of these suggest that the breakage degree is greater in section IIa than the whole.

Two reasons could explain this phenomenon. First, there are the largest relative displacement and compaction degree in this section. Second, on above basis, shifts of caved rocks induce tensile-shear failure first in this section [36].

The change of rock breakage region (binary images in Figure 7) is largely consistent with the movement of curve peak in Figure 6. The breakage region shown in Figure 7 and the peak range of relative displacement in Figure 6 are approximately equal. These suggest that the largest relative displacement and compression degree are associated with breakage concentration in section IIa.

On the other hand, in order to observe shifts in sample's interior in the second stage, displacement curves with a shorter time interval were given by DIC technology in Figure 8 . And Figure 8 shows the displacement changes around the compression pressure of $8.4 \mathrm{MPa}$.

In Figure 8, the curves could be divided into three sections obviously and be consistent with the trend in Figure 5(b). However, these curves show new characteristic with obvious fluctuation in section IIa of the curves. These fluctuations represent the difference of displacement of adjacent caved rocks and the position between peaks and troughs signify existence of local compression [21]. Locations of these fluctuations are situated in section IIa and consistent with the positions of breakage shown in Figure 7. This is because the movements of rock pieces near both ends are restricted. And largest shifts and compression degree in section IIa induce tensile-shear failure. Thence, the breakage occurs mainly in section IIa of the sample first.
3.4. Implications. According to the stratification characteristics of breakage, there are smaller grain sizes in section IIa comparing with other sections. Breakage could improve the distribution of caved rocks and reduce the permeability [35]. Stratification of breakage in sample's interior results in the stratification of permeability. Thence, permeability of entire sample is determined by the location which has the smaller grain size.

Permeability of different section in the sample's interior could be calculated though the fractal theory and the KozenyCarman equation. The fractal dimension of caved rocks is around 2.5. Under the compression, the fractal dimension increases with the increase of pressure [17]. Thence, this stratification effect could be calculated by the following formulas.

The permeability can be calculated according to the Kozeny-Carman equation [38]:

$$
K=\frac{\Phi^{3}}{c(1-\Phi)^{2} s^{2}},
$$

where $c$ and $s$ are Kozeny-Carman constant and specific surface area, respectively, and $\Phi$ is total porosity in some section.

Porosity of caved rocks can be calculated according to the following formula [10]:

$$
\Phi=\left(1-\frac{\Omega_{\min }}{\Omega_{\max }}\right)^{3-D_{F}},
$$

where $\Omega_{\min }$ and $\Omega_{\max }$ are the minimum particle diameter and the maximum particle diameter respectively and $D_{F}$ is the fragmentation fractal dimension in some section.

This stratification effect should be considered in the research of gas migration and seepage researches of caved rocks and similar medium. Particularly, the stratification of breakage in the second stage of compression would change the permeability a lot. Thence, gas migration and seepage researches considering the difference in different section could be more interesting than considering the sample as a whole. Due to the strength and hardness limitation of the observation window, the experiment only discussed the shallow longwall gob, the burial depth of which is less than approximate $400 \mathrm{~m}$. However, similar breakage phenomena have been observed in control trails, the maximum compression pressure of which has reached $30 \mathrm{MPa}$.

\section{Conclusions}

A stratification phenomenon of breakage and movement law of sample was discovered by the uniaxial confined compression experiment and digital image processing technologies. The compression process could be divided into two stages according to the trend of the stress-strain curve and breakage in a certain pressure range. And along the loading direction, the sample could be divided into three sections according to the behaviors of caved rocks.

Movement laws of sample' interior are different in different stages and sections. As a result, the compression degree is uneven distribution in sample' interior and the maximum compression degree appears in section IIa. Boundary effect 
and changes of voids and contact pressure among caved rocks lead to different movement law in different position in sample's interior. In section I, the boundary effect of the fixed end is the dominant factor, while changes of voids and contact pressure are the dominant factors in section II.

A stratification phenomenon of breakage was discovered, which presents breakage concentration in the middle of the sample (section IIa). In section IIa, the average grain size is smaller than that of the global and has a faster decrease of the average grain sizes. The nonlinear movement and shear dislocation induced by shifts among caved rocks are the reason of the breakage stratification phenomenon. These behaviors of breakage would have an effect on the permeability and seepage research of similar medium.

\section{Competing Interests}

The authors declare that they have no competing interests.

\section{Acknowledgments}

This work was financially supported by the National Natural Science Foundation of China (51422404, 51574172), Key Science and Technology Development Program Based on Coal of Shanxi Province in 2014 (MQ2014-12), and Young and Mid-Aged Outstanding Innovation Talents Program of Shanxi Province.

\section{References}

[1] D. M. Pappas and C. Mark, Behavior of Simulated Longwall Gob Material, US Department of the Interior, Bureau of Mines, 1993.

[2] B. Huang, Q. Cheng, and S. Chen, "Phenomenon of methane driven caused by hydraulic fracturing in methane-bearing coal seams," International Journal of Mining Science and Technology, vol. 26, no. 5, pp. 911-927, 2015.

[3] S. Hu, Z. Li, G. Feng et al., "Changes on methane concentration after $\mathrm{CO}_{2}$ injection in a longwall gob: a case study," Journal of Natural Gas Science and Engineering, vol. 29, pp. 550-558, 2016.

[4] M. Qian and P. Shi, Mining Pressure and Strata Control, China University of Mining and Technology Press, Xuzhou, China, 2003.

[5] S. S. Peng, Coal Mine Ground Control, John Wiley \& Sons Inc, New York, NY, USA, 1978.

[6] G. Feng, J. Zheng, Y. Ren, X. Wang, L. Kang, and H. Liu, "Decision theory and method on feasibility on the upward fully mechanized mining of the left-over coal above gob area mined with caving method," Journal of China Coal Society, no. 11, pp. 1863-1867, 2010.

[7] Y. Liu, S. Shao, X. Wang, L. Chang, G. Cui, and F. Zhou, "Gas flow analysis for the impact of gob gas ventholes on coalbed methane drainage from a longwall gob," Journal of Natural Gas Science and Engineering, vol. 36, pp. 1312-1325, 2015.

[8] H. Yavuz, "An estimation method for cover pressure reestablishment distance and pressure distribution in the goaf of longwall coal mines," International Journal of Rock Mechanics and Mining Sciences, vol. 41, no. 2, pp. 193-205, 2004.

[9] B. Yao, Q. Ma, J. Wei, J. Ma, and D. Cai, "Effect of protective coal seam mining and gas extraction on gas transport in a coal seam," International Journal of Mining Science and Technology, vol. 26, no. 4, pp. 637-643, 2015.

[10] C. Ö. Karacan, "Prediction of porosity and permeability of caved zone in longwall gobs," Transport in Porous Media, vol. 82, no. 2, pp. 413-439, 2010.

[11] D. Ma, X. X. Miao, G. H. Jiang, H. B. Bai, and Z. Q. Chen, "An experimental investigation of permeability measurement of water flow in crushed rocks," Transport in Porous Media, vol. 105, no. 3, pp. 571-595, 2014.

[12] D. Ma, X. X. Miao, Z. Q. Chen, and X. B. Mao, "Experimental investigation of seepage properties of fractured rocks under different confining pressures," Rock Mechanics and Rock Engineering, vol. 46, no. 5, pp. 1135-1144, 2013.

[13] C. Su, M. Gu, X. Tang, and W. Guo, "Experiment study of compaction characteristics of crushed stones from coal seam roof," Chinese Journal of Rock Mechanics and Engineering, vol. 31, no. 1, pp. 18-26, 2012.

[14] E. L. Liu, "Deformation and breakage properties of crushable blocky materials," Rock Mechanics and Rock Engineering, vol. 44, no. 3, pp. 373-377, 2011.

[15] E. Liu, "Deformation mechanisms of crushable blocky materials upon lateral unloading for a biaxial stress state," Rock Mechanics and Rock Engineering, vol. 45, no. 3, pp. 439-444, 2012.

[16] W. Zhou, X. Chang, C. Zhou, and K. Zhu, "Stochastic granule discontinuous deformation model of rockfill and its application," Chinese Journal of Rock Mechanics and Engineering, vol. 28, no. 3, pp. 491-499, 2009.

[17] Y. Xu, Y. Xi, X. Feng, H. Zhu, and F. Chu, "Simulation of Rock Grain Breakage Using PFC ${ }^{2 \mathrm{D}}$," Journal of Engineering Geology, vol. 23, no. 4, pp. 589-596, 2015.

[18] Y. Rozenblat, D. Portnikov, H. Kalman, S. Aman, and J. Tomas, "Strength of particles under compression," in Proceedings of the 6th International Conference for Conveying and Handling of Particulate Solids, Engineers Australia, p. 484, Brisbane Convention \& Exhibition Centre, Queensland, Australia, August 2009.

[19] Y. Xiao, Y. Sun, and K. F. Hanif, "A particle-breakage critical state model for rockfill material," Science China Technological Sciences, vol. 58, no. 7, pp. 1125-1136, 2015.

[20] E. Alaei and A. Mahboubi, "A discrete model for simulating shear strength and deformation behaviour of rockfill material, considering the particle breakage phenomenon," Granular Matter, vol. 14, no. 6, pp. 707-717, 2012.

[21] G. Ma, W. Zhou, and X.-L. Chang, "Modeling the particle breakage of rockfill materials with the cohesive crack model," Computers and Geotechnics, vol. 61, pp. 132-143, 2014.

[22] Y. Zhao, J. Zhao, D. Shi, Z. Feng, W. Liang, and D. Yang, "MicroCT analysis of structural characteristics of natural gas hydrate in porous media during decomposition," Journal of Natural Gas Science and Engineering, vol. 31, pp. 139-148, 2016.

[23] S. Zhang, P. Yang, P. Jiang, and T. Zhang, "Determination of initial gas saturation in tight gas formations with NMR relaxometry measurements," Journal of Natural Gas Science and Engineering, vol. 27, pp. 1512-1517, 2015.

[24] Z. Xu, Q. Teng, X. He, and Z. Li, "A reconstruction method for three-dimensional pore space using multiple-point geology statistic based on statistical pattern recognition and microstructure characterization," International Journal for Numerical and Analytical Methods in Geomechanics, vol. 37, no. 1, pp. 97-110, 2013.

[25] X. Liu, Z. Wang, Y. Fu, W. Yuan, and L. Miao, "Macro/ microtesting and damage and degradation of sandstones under 
dry-wet cycles," Advances in Materials Science and Engineering, vol. 2016, Article ID 7013032, 16 pages, 2016.

[26] E.-M. Charalampidou, S. A. Hall, S. Stanchits, G. Viggiani, and H. Lewis, "Shear-enhanced compaction band identification at the laboratory scale using acoustic and full-field methods," International Journal of Rock Mechanics and Mining Sciences, vol. 67, pp. 240-252, 2014.

[27] Q. B. Zhang and J. Zhao, "Determination of mechanical properties and full-field strain measurements of rock material under dynamic loads," International Journal of Rock Mechanics and Mining Sciences, vol. 60, pp. 423-439, 2013.

[28] L. L. Wang, M. Bornert, E. Héripré, S. Chanchole, A. Pouya, and B. Halphen, "The Mechanisms of deformation and damage of mudstones: a micro-scale study combining ESEM and DIC," Rock Mechanics and Rock Engineering, vol. 48, no. 5, pp. 19131926, 2014.

[29] R. A. Salinas, U. Raff, and C. Farfan, "Automated estimation of rock fragment distributions using computer vision and its application in mining," IEE Proceedings-Vision Image and Signal Processing, vol. 152, no. 1, pp. 1-8, 2005.

[30] Y. Tan, Y. Yin, and T. Zhao, "Investigation of rock failure pattern in creep by digital speckle correlation method," Advances in Materials Science and Engineering, vol. 2013, Article ID 916069, 9 pages, 2013.

[31] J.-G. Zhu, H.-Y. Weng, X.-M. Wu, and H.-L. Liu, "Experimental study of compact density of scaled coarse-grained soil," Rock and Soil Mechanics, vol. 31, no. 8, pp. 2394-2398, 2010.

[32] D. Deb and S. Bhattacharjee, "Extended digital image correlation method for analysis of discrete discontinuity," Optics and Lasers in Engineering, vol. 74, pp. 59-66, 2015.

[33] J. Dautriat, M. Bornert, N. Gland, A. Dimanov, and J. Raphanel, "Localized deformation induced by heterogeneities in porous carbonate analysed by multi-scale digital image correlation," Tectonophysics, vol. 503, no. 1-2, pp. 100-116, 2011.

[34] H. Song, H. Zhang, D. Fu, and Q. Zhang, "Experimental analysis and characterization of damage evolution in rock under cyclic loading," International Journal of Rock Mechanics \& Mining Sciences, vol. 88, pp. 157-164, 2016.

[35] Z. Yan, Research on Physical Mechanical Characteristics of Rockfill and Its Engineering Applications, Chongqing University, 2003.

[36] D. Kong, Particle Breakage Strain of Rockfill Materials and Its Numerical Simulation, Tsinghua University, Beijing, China, 2008.

[37] G. Gai, Z. Tao, and M. Ding, Powder Engineering, Tsinghua University Press, Beijing, China, 2009.

[38] P. Xu, S. Qiu, Z. Jiang, and Y. Jiang, "Fractal analysis of Kozeny-Carman constant in the homogenous media," Jounral of Chongqing University (Natural Science Edition), no. 4, pp. 7882, 2011. 

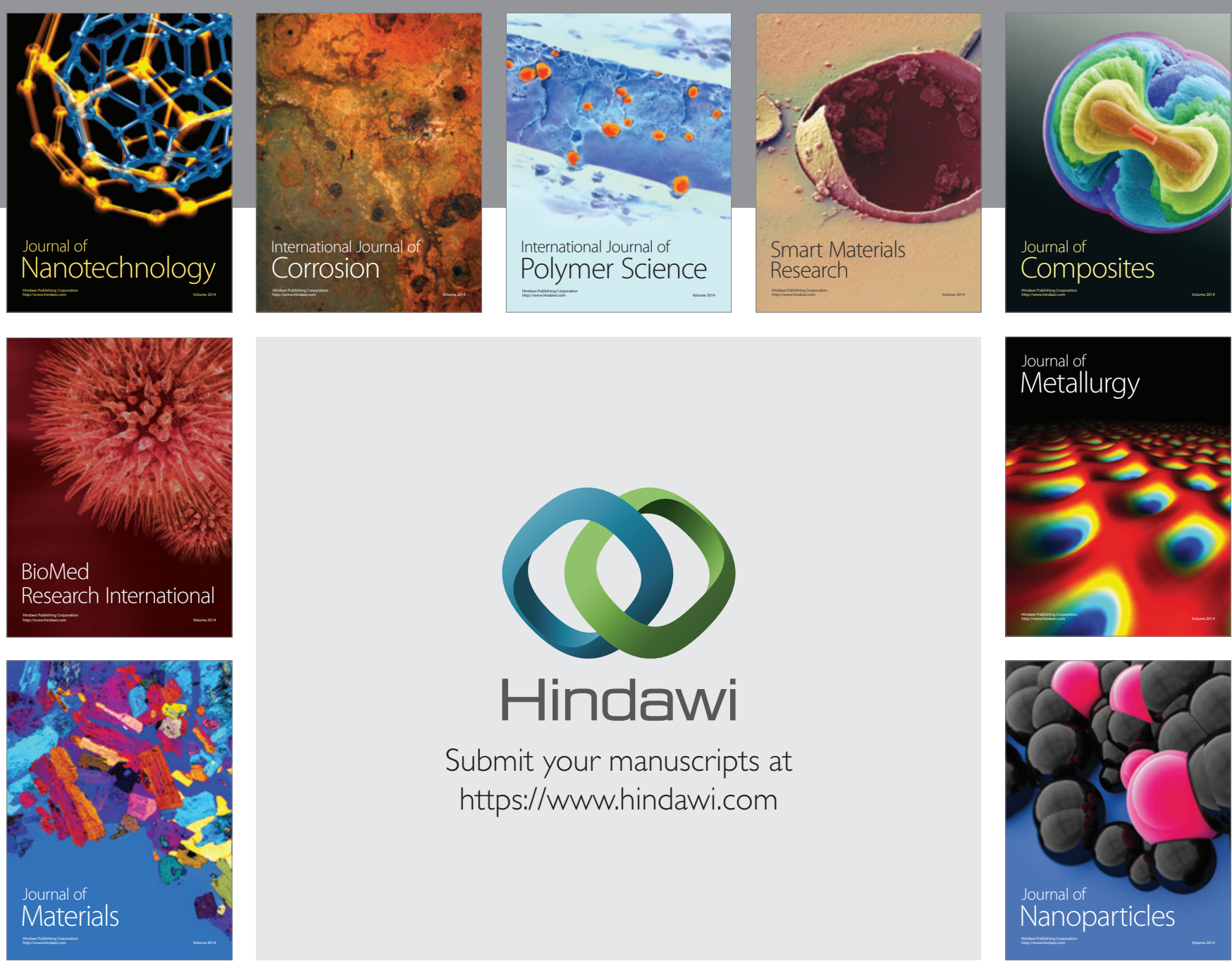

\section{Hindawi}

Submit your manuscripts at

https://www.hindawi.com

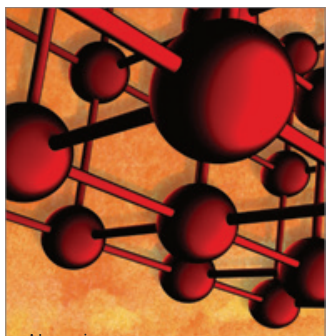

Materials Science and Engineering
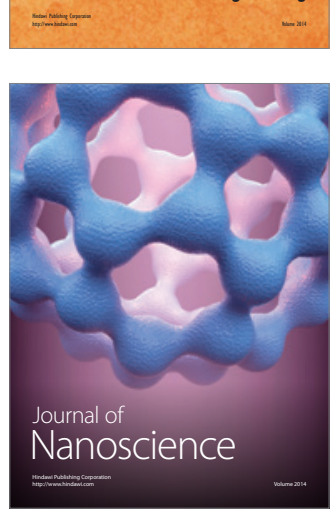
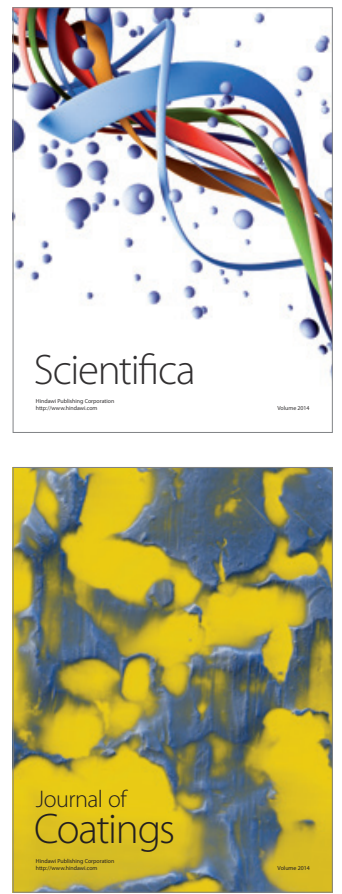
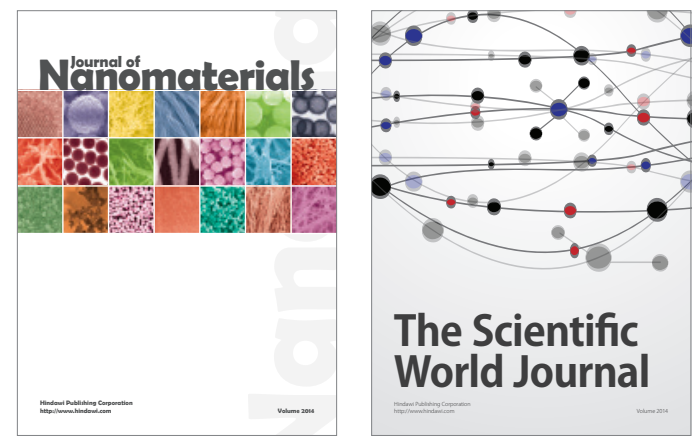

The Scientific World Journal
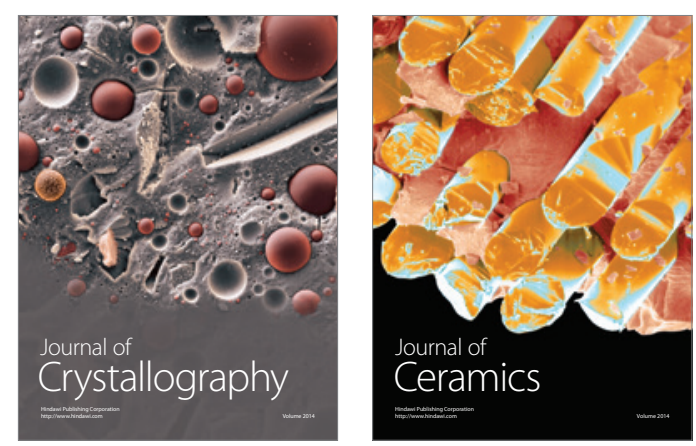
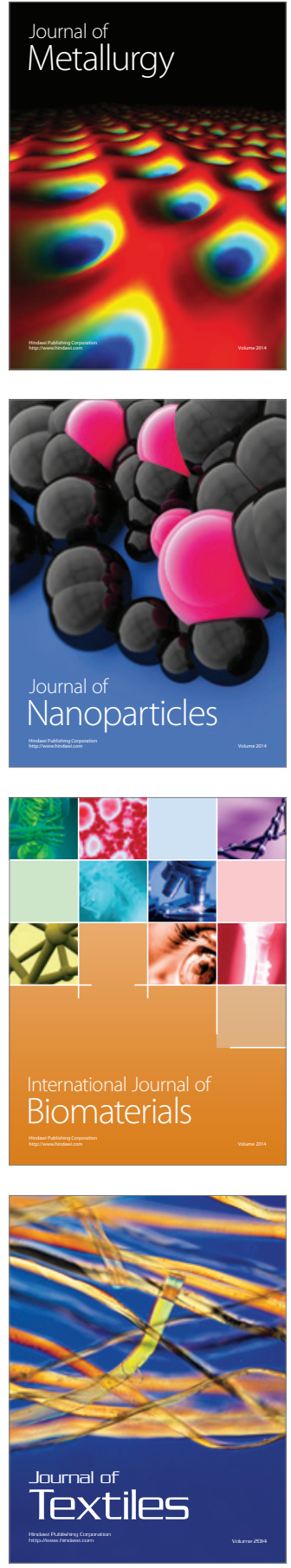